\title{
Novel fine gauge electrocautery dilator for endoscopic ultrasound-guided biliary drainage: experimental and clinical evaluation study (with video) $\square$
}

\section{(ㄷ)(1) $(-2)$}

\author{
Authors \\ Institutions \\ 1 2nd Department of Internal Medicine, Osaka Medical \\ College, Osaka, Japan \\ 2 Department of Gastroenterology, Graduate School of \\ Medicine, The University of Tokyo, Tokyo, Japan \\ 3 First Department of Internal Medicine, Gifu University \\ Hospital, Gifu, Japan \\ 4 Department of Gastroenterology and Hepatology, \\ Tokyo Medical University, Tokyo, Japan
}

Takeshi Ogura ${ }^{1}$, Yosuke Nakai ${ }^{2}$, Takuji Iwashita ${ }^{3}$, Kazuhide Higuchi $^{1}$, Takao Itoi $^{4}$

submitted 18.2.2019

accepted after revision 20.5.2019
Bibliography

DOI https://doi.org/10.1055/a-0961-7890 |

Endoscopy International Open 2019; 07: E1652-E1657

(c) Georg Thieme Verlag KG Stuttgart · New York

eISSN 2196-9736

Corresponding author

Takao Itoi, Department of Gastroenterology and

Hepatology, Tokyo Medical University, 6-7-1 Nishishinjuku,

Shinjuku-ku, Tokyo 160-0023, Japan

Fax: $+81-726846532$

itoitakao@gmail.com
The most important among the steps of endoscopic ultrasound-guided biliary drainage (EUS-BD) is the success of tract dilation. Electrocautery dilation can certainly dilate the biliary or luminal wall. However, bleeding may occur around the dilation site due to burning effects. Here, we describe some technical tips for tract dilation in EUS-BD using a novel electrocautery device. We attempted EUS-BD using the novel electrocautery dilator in 12 patients, among whom nine and three underwent EUS-guided hepaticogastrostomy and EUS-guided gallbladder drainage, respectively. Severe adverse events were not seen in any patients although abdominal pain, which might occur due to stent expansion, was seen in one patient. The novel electrocautery dilator could be inserted into the biliary tract and gallbladder after guidewire placement, and the stent delivery system could also be inserted without additional dilation. Therefore, technical success was achieved in all patients. The procedural durations were less than 20 minutes for each technique. In conclusion, the novel electrocautery dilator might be useful as a tract dilation device, although additional cases and prospective evaluation studies are essential to confirm our results.

\section{Introduction}

Endoscopic ultrasound-guided biliary drainage (EUS-BD) has emerged as an alternative drainage technique for endoscopic retrograde cholangiopancreatography (ERCP) [1]. EUS-BD can be divided into two main categories. The first category is the transduodenal route, for example, EUS-guided choledochoduo- denostomy (CDS). The second is transgastric, for example, EUSguided hepaticogastrostomy (HGS). In addition, EUS-guided gallbladder drainage (GBD) can be performed from both routes. Among these procedures, technical tips can be divided into four steps. The first category is puncturing the biliary tract. The second is guidewire insertion, followed by tract dilation. The final step is stent deployment. Successful tract dilation is the most important among these steps.

To date, various techniques have been reported for tract dilation including graded, balloon, or electrocautery dilation [2 -4]. To prevent bile leakage during EUS-BD, a simple dilation technique may be used. The electrocautery dilation technique can certainly dilate the biliary or luminal wall; however, bleeding may occur around the dilation site due to its burning effects. To overcome this, we have developed a novel fine gauge electrocautery dilator device [5]. An experimental study was performed before clinical use of this novel electrocautery dilator.

In this technical view, we describe the results of our experimental study and some technical tips for tract dilation in EUS$\mathrm{BD}$ using this novel electrocautery dilator.

\section{Patients and method}

\section{Novel fine gauge electrocautery dilator and experimental study}

- Fig. 1 shows the novel electrocautery dilator (Fine 025, Medico's Hirata Inc., Osaka, Japan) (\ Fig.1a). In the experimental study of the burning effect, we compared a conventional elec- 


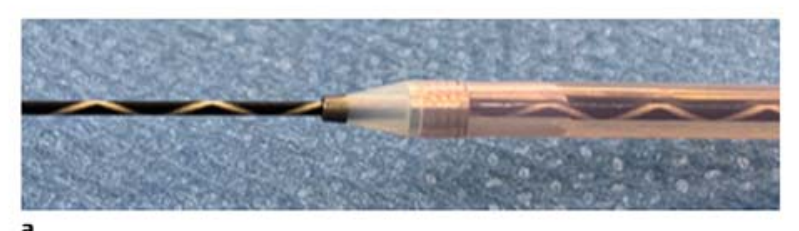

a

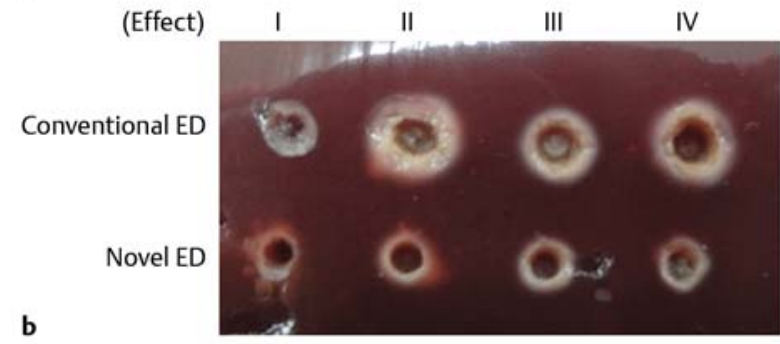

- Fig. 1 a The novel fine gauge electrocautery dilator (Fine 025, Medico's Hirata Inc., Japan, Osaka). The distal end of the outer dilator contains a metal tip, and the top of this metal tip is only $3 \mathrm{Fr}$. This electrocautery dilator is wire-guided, coaxial with the 0.025 inch guidewire. $\mathbf{b}$ Image of the tract and burning effect. Compared with a conventional electrocautery dilator, the burning effect of the novel electrocautery dilator is smaller at each effect setting.

trocautery dilator (6 Fr, Cysto-Gastro-Set; Endo-Flex GmbH, Voerde, Germany) with the novel electrocautery dilator ( $\triangleright$ Table 1), using resected fresh liver from adult pigs. Energy was delivered by an electronic generator. The Erbe VIO300 D electrosurgical system (Erbe Elektromedizin GmbH, Tubingen, Germany) was used for electrocautery with Auto-Cut mode; effect 1 to $4(550 \mathrm{~V})$ was set, and electrocautery dilation was performed in around 2 seconds. The diameter of the tract and the tract including the burning effect were measured, and the differences in burning effect between the two devices were compared.

\section{Patients}

This retrospective study was carried out in Osaka Medical College between June 2018 and November 2018. The inclusion criteria comprised obstructive jaundice or acute cholecystitis, having failed an ERCP, or inaccessible ampulla of Vater due to malignant duodenal obstruction or surgically altered anatomy such as Rouen-Y anastomosis. The exclusion criteria comprised being contraindicated for endoscopic biliary drainage including ERCP and EUS guidance due to conditions such as massive ascites, or performance status determined as Eastern Cooperative Oncology Group (ECOG) scores of 3 or 4 , other organ failure, or withholding consent.

\section{Technical tips for EUS-BD using the novel electrocautery dilator}

All procedures were implemented by endoscopists who were trained and experienced in diagnostic and therapeutic procedures under ERCP guidance at the participating institutions. Patients received antibiotics before undergoing all procedures under sedation.
Table 1 Results of the experimental study.

\begin{tabular}{|c|c|c|c|}
\hline & Effect & Tract, mm & $\begin{array}{l}\text { Tract including } \\
\text { burning effect, mm }\end{array}$ \\
\hline Conventional ED & 1 & 1.57 & 2.54 \\
\hline Novel ED & & 0.80 & 1.60 \\
\hline Conventional ED & 2 & 1.68 & 2.66 \\
\hline Novel ED & & 1.14 & 1.76 \\
\hline Conventional ED & 3 & 1.78 & 2.71 \\
\hline Novel ED & & 1.26 & 1.98 \\
\hline Conventional ED & 4 & 1.81 & 2.98 \\
\hline Novel ED & & 1.37 & 1.95 \\
\hline ED, electrocautery dilator. & & & \\
\hline
\end{tabular}

- Fig. 2 shows the EUS-HGS procedure. A convex EUS (GFUCT240 or 260; Olympus Optical, Tokyo, Japan) connected to an ultrasound device (SSD5500; Aloka, Tokyo, Japan) was initially advanced into the intestine to visualize the intrahepatic bile duct, which was then punctured using a 19G needle (Sono Tip Pro Control, Medi-Globe GmbH, Germany) via color Doppler ultrasonography to avoid intervening vessels. After aspirating the bile juice, the contrast medium was injected ( $\triangleright$ Fig. 2a). Then, a 0.025-inch guidewire (Olympus Medical Systems, Tokyo, Japan) was inserted into the biliary tract ( $\boldsymbol{F}$ Fig. $\mathbf{2 b}$ ). Next, the tract was dilated using the novel electrocautery dilator (effect 4) ( $\triangleright$ Fig.2c). Finally, an 8.5-Fr stent delivery system (Niti-S Biliary Covered Metal Stent; TaeWoong Medical, Seoul, Korea) was inserted into the intrahepatic bile duct, and stent deployment was performed ( $\boldsymbol{F}$ Fig. $2 \mathbf{d}$ ). To prevent stent migration into the abdominal cavity in EUS-HGS, a $10 \mathrm{~cm}$ length of the metal stent was used in all patients.

- Video 1 shows the EUS-GBD procedure. The neck of the gallbladder was punctured using a 19G FNA needle. After the bile juice had been aspirated, the 0.025 -inch guidewire was inserted into the gallbladder. Next, the novel electrocautery dilator was inserted, and the duodenum and gallbladder wall were dilated. After this procedure, an 8-Fr stent delivery system (BONA Biliary Covered Metal Stent; Standard Sci Inc., Seoul, South Korea) was inserted into the gallbladder, and stent deployment was performed from the gallbladder to the duodenum. In EUS-GBD, a $6 \mathrm{~cm}$ length of the metal stent was used in all patients.

Finally, if these stent delivery systems could not be inserted during both procedures, another dilation technique such as balloon dilation was used.

\section{Definition}

The primary end point of this study was focused on adverse events, and technical success was evaluated secondarily. Technical success was defined as successful metal stent deployment after tract dilation using only the novel electrocautery dilator. In addition, clinical success of EUS-HGS was defined as a de- 

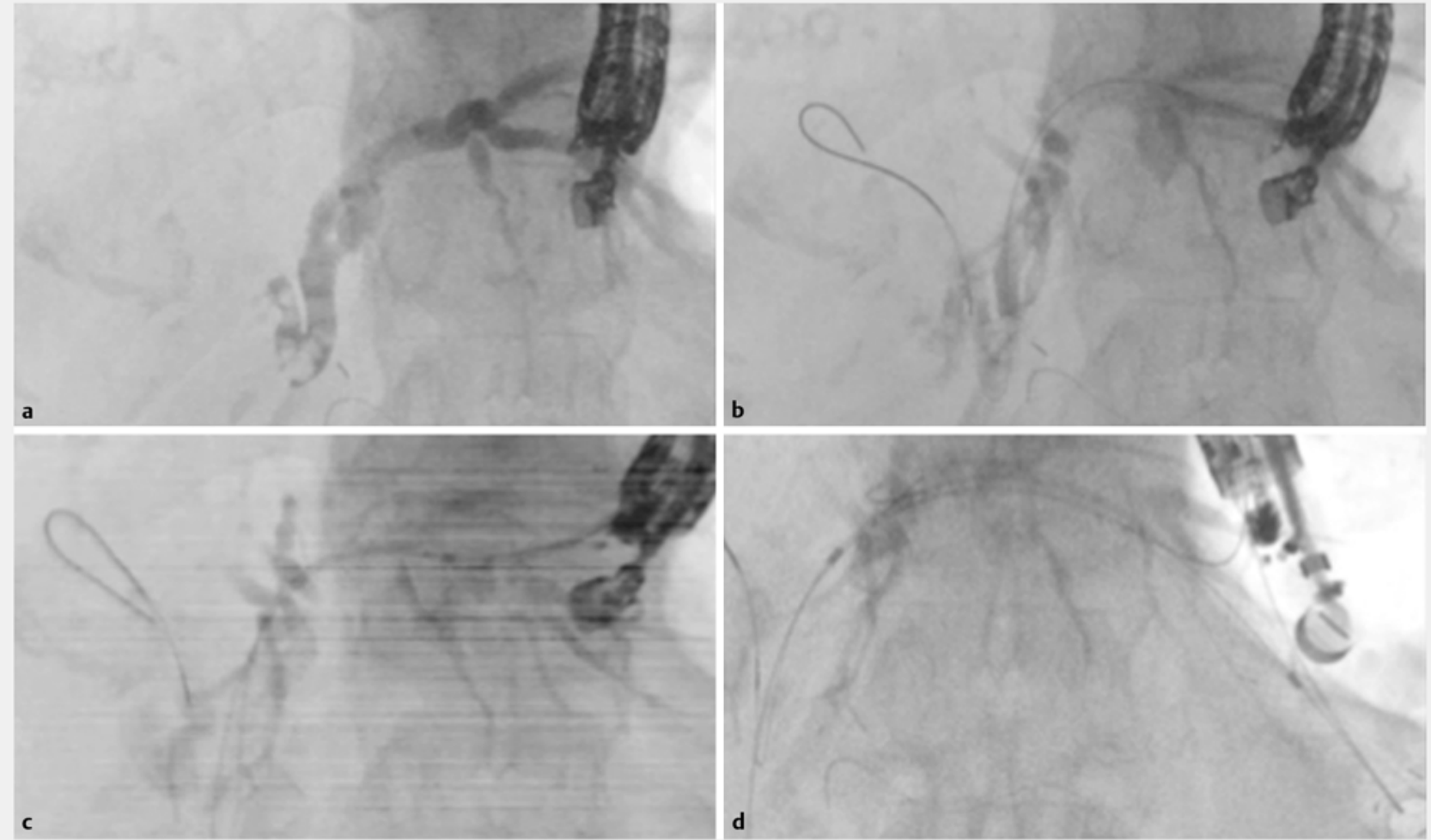

- Fig. 2 a The contrast medium is injected after the intrahepatic bile duct is punctured using a $19 \mathrm{G}$ needle. $\mathbf{b}$ The 0.025 -inch guidewire is inserted into the biliary tract. c The bile duct wall is dilated using the novel electrocautery dilator. d Covered self-expandable metal stent deployment is performed from the intrahepatic bile duct to the stomach.

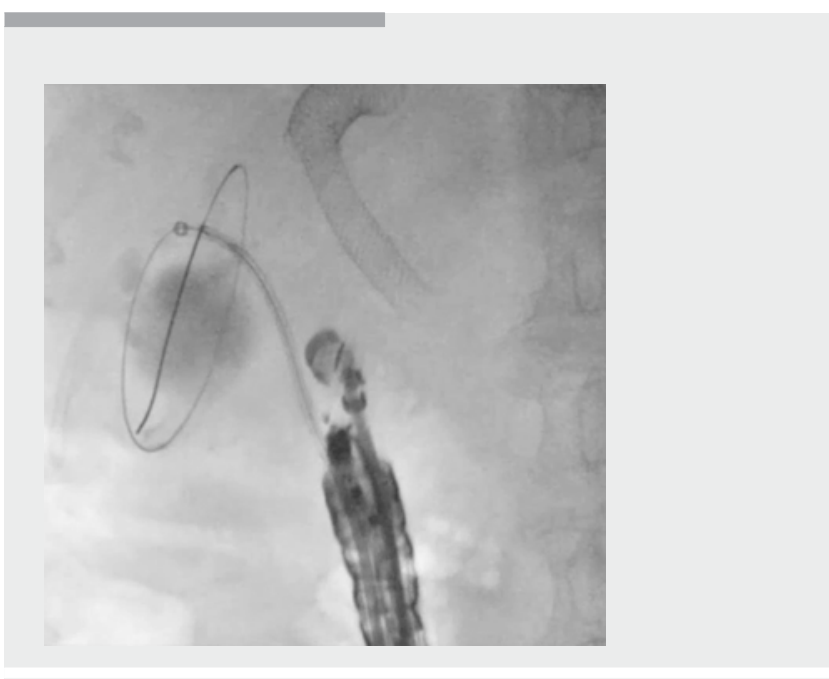

Video 1 Endoscopic ultrasound-guided gallbladder drainage. The gallbladder is punctured using a $19 \mathrm{G}$ needle, and the contrast medium is injected. The 0.025 -inch guidewire is inserted into the gallbladder. Then, the duodenal and gallbladder walls are dilated using the novel electrocautery dilator. Finally, stent deployment is successfully performed from the gallbladder to the duodenum. ease in serum bilirubin to $<75 \%$ of pre-biliary drainage within 30 days. Clinical success of EUS-GBD was defined as complete resolution of clinical symptoms, such as abdominal pain and fever, or decreased inflammation on blood tests.

Adverse events including bleeding, perforation, and stent migration during the procedure were evaluated. Bleeding was defined as that necessitating transfusion or requiring hospitalization, upper endoscopy, or a procedure by interventional radiology. Adverse events were graded according to the severity grading system of the American Society for Gastrointestinal Endoscopy [6]. Adverse events occurring with 1 month after the procedure and possibly related to it were described in accordance with this guideline. Procedure time was measured from puncturing of the target lesion to stent deployment.

\section{Results}

\section{Experimental study}

Differences between the conventional and novel electrocautery dilator were examined. As shown in > Fig. 1b, the burning effect of the novel electrocautery dilator was visibly smaller compared with the conventional electrocautery dilator. Indeed, among effects 1 to 4 , the diameter of the tract including the burning effect was smaller with the novel electrocautery dilator ( Table 1). 
- Table 2 Patient characteristics.

\begin{tabular}{|c|c|c|c|c|c|c|}
\hline No. & Age/Sex & Disease & Procedure & Technical success & Adverse events & Procedure time, $\min$ \\
\hline 1 & $78 / \mathrm{M}$ & $A C$ & GBD & Yes & None & 12 \\
\hline 2 & $81 / F$ & PC & HGS & Yes & None & 18 \\
\hline 3 & $70 / \mathrm{M}$ & PC & HGS & Yes & None & 16 \\
\hline 4 & $70 / \mathrm{F}$ & PC & HGS & Yes & None & 12 \\
\hline 5 & $69 / F$ & PC & HGS & Yes & Abdominal pain & 11 \\
\hline 6 & $73 / \mathrm{M}$ & $A C$ & GBD & Yes & None & 10 \\
\hline 7 & $81 / \mathrm{M}$ & $\mathrm{BC}$ & HGS & Yes & None & 13 \\
\hline 8 & $78 / \mathrm{M}$ & $A C$ & GBD & Yes & None & 16 \\
\hline 9 & $88 / \mathrm{M}$ & GC & HGS & Yes & None & 22 \\
\hline 10 & $81 / \mathrm{M}$ & GC & HGS & Yes & None & 19 \\
\hline 11 & $72 / F$ & GC & HGS & Yes & None & 20 \\
\hline 12 & $77 / \mathrm{M}$ & PC & HGS & Yes & None & 17 \\
\hline
\end{tabular}

M, male; F, female; PC, pancreatic cancer; BC, bile duct cancer; AC, acute cholecystitis; GC, gastric cancer; HGS, hepaticogastrostomy; GBD, gallbladder drainage.

\section{Clinical study}

EUS-BD was attempted in 12 patients using the novel electrocautery dilator ( $\triangleright$ Table 2 ). Among the 12 patients, EUS-HGS was performed because of surgical anatomy $(n=10)$, or duodenal obstruction $(n=2)$, and three patients underwent EUS-GBD as their condition was either too poor to undergo surgical treatment $(n=2)$, or they had advanced cancer $(n=1)$. An antithrombotic agent (warfarin potassium) was administered for one patient who underwent EUS-HGS.

For the EUS-HGS procedure, the diameter of the intrahepatic bile duct was around $4 \mathrm{~mm}$, and the puncture site was B3 in all patients. Severe adverse events including bile peritonitis were not seen in any patient although abdominal pain, which might occur due to the stent expanding, was seen in one patient. The novel electrocautery dilator could be inserted into the biliary tract and gallbladder after guidewire placement, and the stent delivery system could also be inserted without any dilation device apart from the diathermic dilator. Therefore, technical and clinical success was achieved in all patients. The procedure time was less than 22 minutes (range 11 to 22).

Among the three patients who underwent EUS-GBD, the etiology of acute cholecystitis was gallbladder stones $(n=2)$, and cystic duct obstruction due to pancreatic cancer $(n=1)$. All EUS-GBD procedures were performed from the duodenum. After tract dilation using the novel electrocautery dilator, stent deployment was successfully performed in all patients without an additional dilation technique. Clinical success was achieved in all patients without any adverse events. The procedure time was within 16 minutes (range 10 to 16 ).

\section{Discussion}

According to a recent review of EUS-BD which included 686 patients undergoing either HGS or CDS, the overall technical and clinical success rates were, respectively $96 \%(95 \% \mathrm{Cl}: 93-98)$ and $84 \%$ (95\%Cl: $80-88)$ for HGS, and, respectively $95 \%(95 \%$ Cl: 91 - 97) and $87 \%$ (95\%Cl: 82 - 91) for CDS. However, the rate of adverse events such as bleeding, hemobilia, peritonitis, and pneumoperitoneum was relatively high in $29 \%$ of patients undergoing HGS, and $20 \%$ of patients undergoing CDS [7]. In a review of EUS-GBD [8], overall technical success was achieved in $83.7 \%(185 / 221)$, and clinical success in $92.3 \%(204 / 221)$ of patients. Procedure-related adverse events were seen in 16 patients (7.2\%). Pneumoperitoneum was the most commonly seen adverse event $(53 \%, n=9)$. If EUS-GBD was performed using a lumen apposing metal stent, overall technical success was achieved in $90.2 \%$ (259/287), and clinical success in $93.0 \%$ (267/287) of patients. Procedure-related adverse events were seen in 23 patients $(8.0 \%)$.

Almost all of these adverse events can be managed conservatively. However, EUS-BD is sometimes attempted in patients with poor condition, therefore, these adverse events may decrease the quality of life and may sometimes be fatal. In addition, in terms of being able to undergo chemotherapy or recommencing solid food intake without a delay, avoiding these adverse events can have a clinical impact.

Bile peritonitis may occur during the EUS-BD procedure, especially during device exchanging. Therefore, a simplified technique may be preferred during EUS-BD procedures. Among the steps in EUS-BD procedures, tract dilation should be simplified. Paik et al. reported a simplified tract dilation technique for EUS-HGS [2]. They used a balloon catheter with a 4-Fr tip to dilate the tract. The technical success rate was $96 \%$ (27/28), and median procedure time was short (15.3 minutes); however, one 

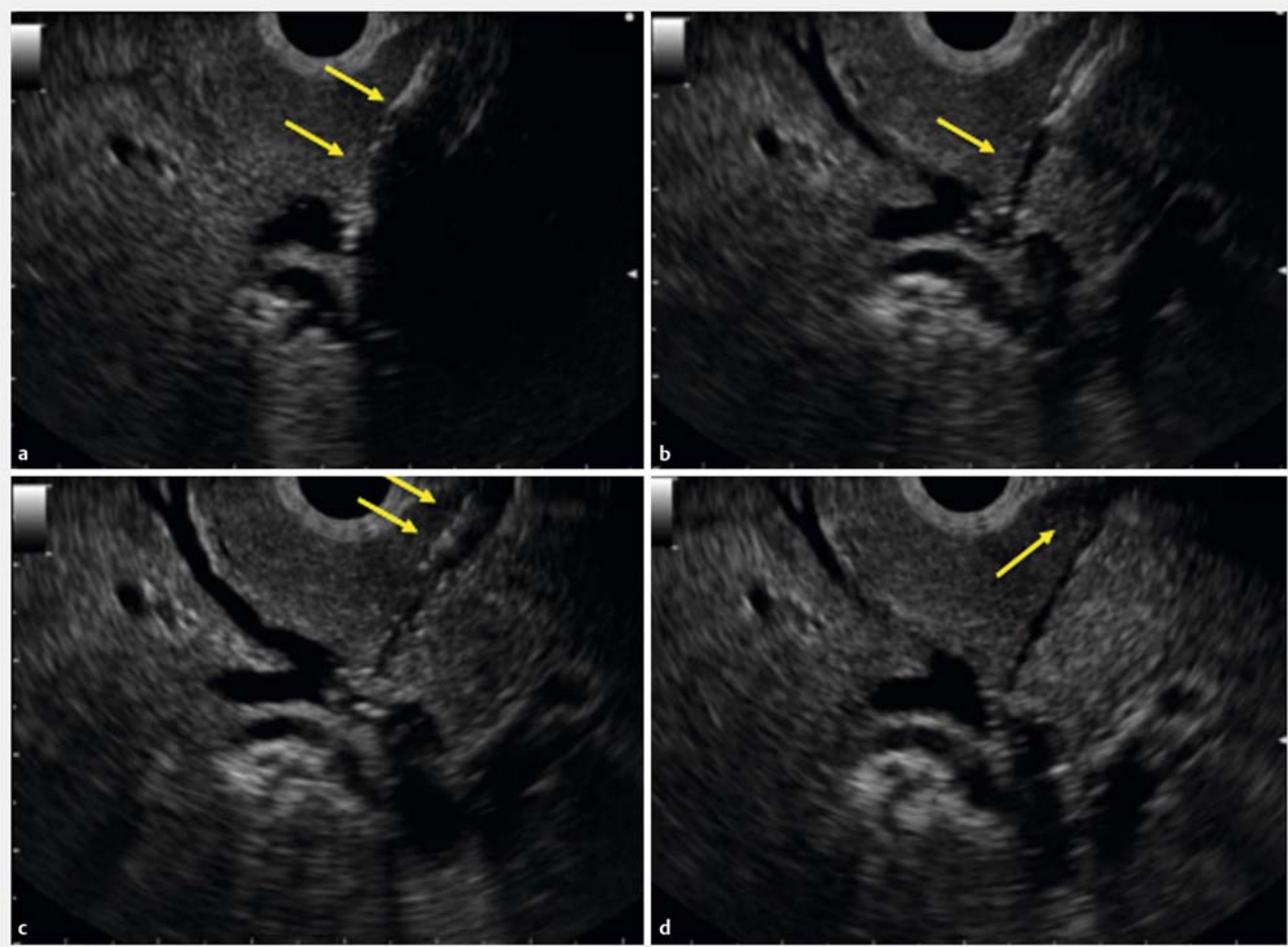

- Fig. 3 a The intrahepatic bile duct is dilated using a balloon catheter. $\mathbf{b}$ The hole is made by balloon dilation. $\mathbf{c}$ The stomach wall is dilated using the balloon catheter. $\mathbf{d}$ Bile leakage is seen because the hepatic parenchyma is dilated by the balloon catheter.

patient needed fistula dilation using a needle-knife. In addition, to manipulate the guidewire in place in the desired intrahepatic bile duct, a 4-Fr cannula was initially used in seven patients. We performed a prospective clinical study of EUS-BD using a novel fine gauge balloon catheter [3]. In that study, technical success was defined as successful stent deployment after tract dilation using only the balloon catheter (diameter of this balloon was 3 $\mathrm{Fr}$ ). As result, technical success was achieved in all patients including those undergoing EUS-CDS and HGS.In addition, adverse events were seen in self-limited abdominal pain $(n=2)$, and bile peritonitis $(n=1)$. Therefore, this device may have clinical benefit as a simplified dilation technique; however, as shown > Fig. 3, the balloon catheter dilated not only the bile duct and luminal wall but also the hepatic parenchyma, therefore, bile leakage can easily occur through this tract.

On the other hand, the electrocautery dilation technique can certainly dilate only the bile duct and transluminal wall, and compared with balloon dilation, the frequency of bile leakage may be reduced; however, the electrocautery dilation technique also has disadvantages such as the risk of bleeding due to the burning effect. Park et al. [4] reported that, among 57 con- secutive patients, post-procedural adverse events occurred in 11 (bile peritonitis, $\mathrm{n}=2$; mild bleeding, $\mathrm{n}=2$; and self-limited pneumoperitoneum, $n=7$ ). In multivariate analysis, using a needle-knife was the only risk factor for post-procedural adverse events in EUS-BD. They concluded that a needle-knife should not be used as a dilation device if possible. Honjo et al. evaluated the ultra-tapered mechanical dilator for EUS-HGS and EUS-guided pancreatic duct drainage compared with the electrocautery dilator [9]. Among 64 patients, 49 underwent EUS-HGS and of these, 23 underwent EUS-HGS with the conventional electrocautery dilator, and 26 patients with a mechanical dilator. Although overall adverse events were also significantly different between the groups, bleeding was frequently observed in the electrocautery dilator group $(P=0.04)$. In that retrospective study, the authors mentioned that the electrocautery dilation technique can cause possible burning effects to the hepatic parenchyma and vessels around the needle tract or the gastrointestinal lumen, causing unexpected bleeding or inflammation. On the other hand, in our study, bleeding was not seen in any patient. During the EUS-BD procedure, a pushing force is also important to insert the various devices 
into the biliary tract. However, if the devices are difficult to advance due to inflammations or wall thickness, electrocautery dilation may be useful because the bile duct wall is certainly dilated, and the procedure time is reduced. In our study, to reduce the cautery effect, the function of the electrocautery dilator was only used when the bile duct and intestinal wall were dilated. In addition, a fine gauge electrocautery dilator was used in EUS-BD procedures. Therefore, EUS-BD using our device and technique may be technically feasible and safe, although there are critical limitations such as the small number of patients in our study, its retrospective nature, and the fact that it was a single-arm trial.

In our experience, the best indication of tract dilation using the novel electrocautery dilator may be the intrahepatic bile duct access such as EUS-HGS or EUS-guided jejunostomy. Regarding EUS-GBD, recently, the electrocautery-enhanced delivery system (hot AXIOS stent, Boston Scientific, Marlborough, Massachusetts, United States) has become available [10]. This stent is a through-the-scope LAMS mounted on a stent delivery system with an electrocautery wire at the distal tip. The electrocautery tip allows passage of the catheter into the gallbladder without the need for prior dilation of the tract by application of a pure cutting current. This fact may have clinical benefits, such as shortening of the procedure time, reduced bile leakage during fistula dilation, and improved technical success rate due to its being a single-step procedure. Although technical tips for EUS-GBD using this stent are simple, and may be easily compared with a conventional stent, this stent is not available in all countries. Therefore, our device may be useful for EUS-GBD using the conventional technique.

In conclusion, the novel electrocautery dilator may be useful as a tract dilation device, but additional cases and a prospective evaluation study are essential to confirm our results.
Competing interests

None

\section{References}

[1] Giovannini M, Dotti M, Bories E et al. Hepaticogastrostomy by echoendoscopy as a palliative treatment in a patient with metastatic biliary obstruction. Endoscopy 2003; 35: 1076-1078

[2] Paik WH, Park DH, Choi JH et al. Simplified fistula dilation technique and modified stent deployment maneuver for EUS-guided hepaticogastrostomy. World J Gastroenterol 2014; 20: 5051-5019

[3] Amano M, Ogura T, Onda S et al. Prospective clinical study of endoscopic ultrasound-guided biliary drainage using novel balloon catheter (with video). J Gastroenterol Hepatol 2017; 32: 716-720

[4] Park DH, Jang JW, Lee SS et al. EUS-guided biliary drainage with transluminal stenting after failed ERCP: predictors of adverse events and long-term results. Gastrointest Endosc 2011; 74: 1276-1284

[5] Ogura T, Nakai Y, Itoi T. Novel fine gauge electrocautery dilator for endoscopic ultrasound-guided hepaticogastrostomy (with video). J Hepatobiliary Pancreat Sci 2019; 26: E3 - E4

[6] Cotton PB, Eisen GM, Aabakken L et al. A lexicon for endoscopic adverse events: Report of an ASGE workshop. Gastrointest Endosc 2010; 71: $446-454$

[7] Hedjoudje A, Sportes A, Grabar S et al. Outcomes of endoscopic ultrasound-guided biliary drainage: A systematic review and meta-analysis. United Eur Gastroenterol J 2019; 7: 60-68

[8] Ogura T, Higuchi K. Endoscopic ultrasound-guided gallbladder drainage: Current status and future prospects. Dig Endosc 2019; 31 : (Suppl. 01): 55-64

[9] Honjo M, Itoi T, Tsuchiya T et al. Safety and efficacy of ultra-tapered mechanical dilator for EUS-guided hepaticogastrostomy and pancreatic duct drainage compared with electrocautery dilator (with video). Endosc Ultrasound 2018; 7: 376-382

[10] Walter D, Teoh AY, Itoi T et al. EUS-guided gall bladder drainage with a lumen-apposing metal stent: a prospective long-term evaluation. Gut 2016; 65: 6-8 